\title{
Free Energy of Solvation, Interaction, and Binding of Arbitrary Charge Distributions Imbedded in a Dielectric Continuum
}

\author{
B. Jayaram \\ Department of Chemistry, Indian Institute of Technology, Hauz Khas, New Delhi-110016, India
}

Received: September 28, 1993; In Final Form: February 17, $1994^{\circ}$

\begin{abstract}
A theoretical treatment of solvation and interaction of two arbitrary charge distributions imbedded in a dielectric continuum solvent is presented. The electrostatic component of the total free energy of such a charge assembly contains contributions from the solvation energies of each charge distribution, and modified Coulomb interaction energies between the two charge distributions. The theory developed is illustrated for special cases along with an application to the interaction between two ions in a continuum solvent.
\end{abstract}

\section{Introduction}

The dielectric continuum treatment of solvent in theoretical descriptions of equilibrium properties of aqueous solutions of simple ions, molecules, and biomolecules has received renewed attention in recent years due to its conceptual simplicity and computational expediency. Both analytical and numerical techniques have been exploited to obtain free energies of hydration of solutes, starting from Laplace or Poisson-Boltzmann equations. The stage is now set to tackle problems of binding equilibria with a continuum treatment of solvent. In this article, we present a theoretical treatment of solvation, interaction, and binding of two arbitrary charge distributions imbedded in a dielectric continuum solvent. We have derived expressions for the free energy of interaction between two solutes as mediated by solvent. This amounts to obtaining the electrostatic contribution to the potential of mean force between the solutes, which is not only of interest per se, but also can be used in molecular simulations to incorporate solvent effects. The theory developed is discussed with an application to the interaction between two ions in a continuum solvent.

\section{Background}

Attempts seeking analytical solutions to the hydration free energies of arbitrary charge distributions have a long history. Born's model for ion solvation ${ }^{1-3}$ and Onsager's reaction field approach $^{4}$ to dipolar solvation found a generalization in Kirkwood's formulation ${ }^{5,6}$ of the Helmholtz free energy of an arbitrary charge distribution imbedded in a dielectric continuum solvent containing added salt. Beveridge and Schnuelle ${ }^{7}$ reported a concentric dielectric continuum model which can in principle incorporate several layers of solvent with varying dielectric constants to account for saturation effects in calculating solvation free energies of arbitrary charge distributions with an overall spherical symmetry. This theory was subsequently extended to other geometries. ${ }^{8,9}$ Jayaram and Beveridge included ionic strength effects in the concentric dielectric model ${ }^{10}$ as an extension to Tanford-Kirkwood theory ${ }^{11}$ and compared the dielectric continuum model results on solvation with those based on molecular simulations. ${ }^{12}$ Solvation and salt effects in the stability of globular proteins, for instance, fall within the purview of such theories. ${ }^{6}$ The SATK (static accessibility Tanford-Kirkwood) model, ${ }^{13}$ which uses a simpler treatment of solvent than that of Beveridge and co-workers but an additional depth parameter, has been extensively applied to study protein titration curves, merits and limitations of which are discussed in several places. ${ }^{13,14}$ States and Karplus ${ }^{15}$ developed an electrostatic continuum solvent model to treat hydrogen-exchange behavior in proteins. ${ }^{16}$ Gilson et al. ${ }^{17}$ categorized the different contributions to electrostatic free energies of macromolecules under two sets. The first set

- Abstract published in Advance ACS Abstracts, April 1, 1994. includes all pairwise interactions, viz. coulombic, boundary polarization effect and a pairwise Debye-Huckel term. The second set includes all self-energies. Gilson and Honig ${ }^{18}$ subsequently presented a numerical method to calculate the total electrostatic energy of a macromolecular system. Solvation free energies of DNA-like molecules with an overall cylindrical symmetry in the continuum framework have also been reported. 19 Bashford ${ }^{20}$ has recently summarized the existing theoretical tools to treat electrostatic effects in biological molecules. Molecular simulations on solvation free energies have provided new insights into the success of continuum models. ${ }^{21-23}$ The above theories deal with solvent and/or salt effects on an ion, molecule, or macromolecule. Dielectric continuum theories, in the above vein, for binding and interaction have not progressed beyond Coulomb's law, although numerical results based on simulations and the integral equation method, ${ }^{24}$ the protein dipole Langevin dipole (PDLD) approach, ${ }^{25,26}$ the finite boundary element method, ${ }^{27}$ and finite difference and finite element Poisson-Boltzmann methods ${ }^{18,28-30}$ have been reported. Binding is the next phase in the theoretical approaches.

Consider two arbitrary charge distributions infinitely apart in a vacuum (dielectric constant $\epsilon=1$ ). When these two charge distributions are brought together to a distance $R$, Coulomb's law gives us the interaction energy as a summation over $\left(q_{\mathrm{i}} q_{\mathrm{j}} /\right.$ $\left.r_{i j}\right)$-type terms. Now if these charge distributions held at a distance $R$ are transferred to a dielectric medium (e.g. $\epsilon=80$ ), the interaction energies are still calculated as $\left(q_{\mathrm{i}} q_{\mathrm{j}} / \epsilon r_{\mathrm{ij}}\right)$, treating each charge distribution as a collection of point charges in a continuum solvent. Suppose each of these charge distributions is imbedded in a low dielectric cavity in a continuum solvent. Coulomb's law is not valid for estimating the interaction energies. The free energy of such an assembly contains contributions coming from the solvation of each charge distribution and its interaction with the other charge distribution as mediated by the solvent. To illustrate this further, let a cation of charge $q_{+}$and radius $r_{+}$ represent charge distribution I and an anion of charge $q_{-}$and radius $r_{-}$represent charge distribution II and consider that they are infinitely apart in a dielectric medium. The free energy of such an assembly in the continuum model may be evaluated as a sum of the Born's self-energy terms or the solvation free energy of each ion. Implicit in Born's formulation is the idea that a point charge is imbedded in a low dielectric cavity which in turn is surrounded by a high dielectric medium. Now when these two ions are brought closer from infinity to a distance $R$, the solvation free energy terms are modified and there is an additional contribution due to the interaction between the two charges. Coulomb's law obviously is not valid for estimating the interaction energies between the two ions because of the presence of the dielectric boundaries between the two ions. Also, usage of Coulomb's law is inconsistent with the Born model. A theory is needed which accounts for both solvation and interaction as a 


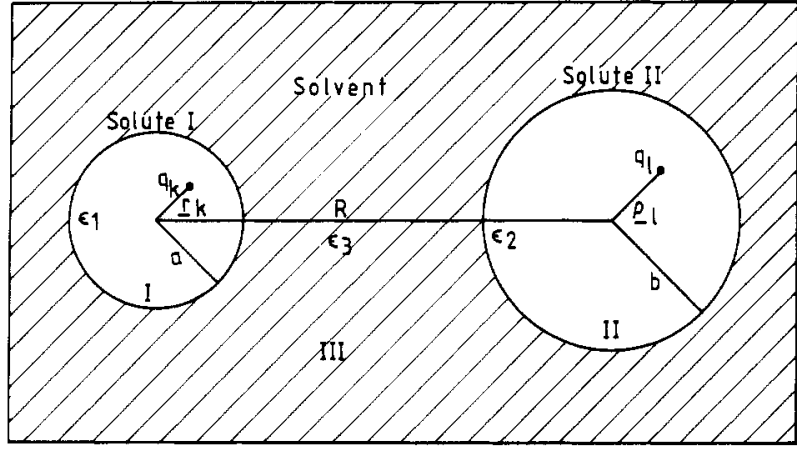

Figure 1. Definition of the parameters for the problem of solvent-mediated interaction of an arbitrary charge distribution enclosed in a sphere of radius $a$ and dielectric constant $\epsilon_{1}$ with another charge distribution enclosed in a sphere of radius $b$ of dielectric constant $\epsilon_{2}$, both separated by a distance $R$ and solvent of dielectric constant $\epsilon_{3}$.

function of distance between the two arbitrary charge distributions. The present paper addresses this need.

\section{Theory and Discussion}

The model considered in this study is depicted in Figure 1. Region I represents a cavity of radius $r=a$ and a dielectric constant $\epsilon_{1}$ where in the solute charges $q_{\mathrm{k}}$ are located at sites $\mathbf{r}_{\mathrm{k}}$. Region II similarly represents a cavity of radius $\rho=b$ and a dielectric constant $\epsilon_{2}$ where in the solute charges $q_{1}$ are located at sites $\mathbf{r}_{1}$. Region III contains solvent of dielectric constant $\epsilon_{3}$. The distance between the centers of the charge distributions of solutes 1 and 2 is denoted as $R$. The objective here is to obtain an analytical expression for the solvation free energies together with the free energy of solvent-mediated interaction between the two charge distributions as a function of distance between them.

To obtain the solvent contribution to the total free energy of the charge assembly, one starts with the Laplace equation

$$
\nabla^{2} \Phi=0
$$

where $\Phi$ is the electrostatic potential and $\nabla^{2}$ is the Laplacian operator. The general solution for eq 1 in polar coordinates is

$$
\Phi=\epsilon^{-1} \sum_{n=0}^{\infty} \sum_{m=-n}^{+n}\left(A_{n m} r^{n}+\frac{B_{n m}}{r^{n+1}}\right) P_{n}^{m}(\cos \theta) \mathrm{e}^{i m \varphi}
$$

where $P_{n}^{m}(\cos \theta)$ are associated Legendre polynomials, $A_{n m}$ is a constant, and $B_{n m}$ is related to the charge distribution.

The problem considered here involves three regions. The potential inside the cavity $I$ is given as

$$
\Phi_{1}=\epsilon_{1}^{-1} \sum_{n=0}^{\infty} \sum_{m=-n}^{+n}\left(A_{n m} r^{n}+\frac{B_{n m}}{r^{n+1}}\right) P_{n}^{m}(\cos \theta) \mathrm{e}^{i m \varphi}
$$

The potential inside cavity II is given as

$$
\Phi_{2}=\epsilon_{2}^{-1} \sum_{n=0}^{\infty} \sum_{m=-n}^{+n}\left(C_{n m} \rho^{n}+\frac{D_{n m}}{\rho^{n+1}}\right) P_{n}^{m}(\cos \theta) \mathrm{e}^{i m \varphi}
$$

The potential in the region of bulk solvent, i.e. region III, is given as

$$
\begin{array}{r}
\Phi_{3}=\epsilon_{3}^{-1} \sum_{n=0}^{\infty} \sum_{m=-n}^{+n}\left(E_{n m} r^{n}+\frac{F_{n m}}{r^{n+1}}+G_{n m} \rho^{n}+\frac{H_{n m}}{\rho^{n+1}}\right) \\
P_{n}^{m}(\cos \theta) \mathrm{e}^{i m \varphi}
\end{array}
$$

The terms containing $\left(1 / r^{n+1}\right)$ and $\left(1 / \rho^{n+1}\right)$ originate in the multipolar expansion of the two charge distributions with " $n$ " denoting the order of the electrical moments. It may be noted that $r, \rho$, and $R$ represent lengths of three sides of a triangle. Let the angle between the sides of length $r$ and $R$ be $\gamma$ and that between $\rho$ and $R$ be $\delta$. Then according to the law of cosines

$$
\begin{aligned}
& \rho^{2}=\left(r^{2}+R^{2}-2 r R \cos \gamma\right) \\
& r^{2}=\left(\rho^{2}+R^{2}-2 \rho R \cos \delta\right)
\end{aligned}
$$

$B_{n m}$ and $D_{n m}$ contain the characteristics of the charge distributions I and II, respectively:

$$
\begin{aligned}
& B_{n m}=\left\{\frac{(n-|m|) !}{(n+|m|) !)}\right\} \sum_{k=1}^{s} q_{k} r_{k}^{n} P_{n}^{m}\left(\cos \theta_{k}\right) \mathrm{e}^{-i m \varphi_{k}} \\
& D_{n m}=\left\{\frac{(n-|m|) !}{(n+|m|) !}\right\} \sum_{l=1}^{t} q_{l} \rho_{l}^{n} P_{n}^{m}\left(\cos \theta_{l}\right) \mathrm{e}^{-i m \varphi_{l}}
\end{aligned}
$$

The terms with $A_{n m}$ and $C_{n m}$ in eqs 3 and 4 are due to the reaction field acting on the solute charge distributions I and II, respectively, originating in the polarization of the bulk dielectric continuum, and these are made to include Coulomb potentials as well, due to charge distributions II and I, respectively, as modified by the presence of dielectric discontinuities by a suitable choice of the (general solutions and) boundary conditions. The reaction as well as the modified Coulomb potential acting on the solute charge distribution I is identifiable with

$$
\Phi_{1}^{R}=\epsilon_{1}^{-1} \sum_{n=0}^{\infty} \sum_{m=-n}^{+n}\left(A_{n m} r^{n}\right) P_{n}^{m}(\cos \theta) \mathrm{e}^{i m \varphi}
$$

Similarly, the reaction as well as the modified Coulomb potential acting on the solute charge distribution II is identifiable with

$$
\Phi_{2}^{R}=\epsilon_{2}^{-1} \sum_{n=0}^{\infty} \sum_{m=-n}^{+n}\left(C_{n m} \rho^{n}\right) P_{n}^{m}(\cos \theta) \mathrm{e}^{i m \varphi}
$$

The Helmholtz free energy of the system is then obtained as

$$
A=\frac{1}{2} \sum_{k=1}^{s} q_{k} \Phi_{1}^{R}\left(r_{k}\right)+\frac{1}{2} \sum_{l=1}^{t} q_{l} \Phi_{2}^{R}\left(\rho_{l}\right)
$$

The above free energy expression contains solvation free energies of each charge distribution and the free energy of interaction between the two charge distributions. The reference state is the two charge distributions infinitely apart in a vacuum or the absence of solvent.

Thus the problem on hand reduces to evaluating the constants $A_{n m}$ and $C_{n m}$ subject to a specification of appropriate boundary conditions. The boundary conditions for the model considered here are that the potential and the dielectric displacement across the boundaries be continuous and the potential be finite in the bulk solvent region.

$$
\begin{gathered}
\Phi_{1(r=a)}=\Phi_{3(r=a)} \\
\Phi_{2(\rho=b)}=\Phi_{3(\rho=b)} \\
\epsilon_{1}\left(\mathrm{~d} \Phi_{1} / \mathrm{d} r\right)_{r=a}=\epsilon_{3}\left(\mathrm{~d} \Phi_{3} / \mathrm{d} r\right)_{r=a} \\
\epsilon_{2}\left(\mathrm{~d} \Phi_{2} / \mathrm{d} \rho\right)_{\rho=b}=\epsilon_{3}\left(\mathrm{~d} \Phi_{3} / \mathrm{d} \rho\right)_{\rho=b} \\
\Phi_{3} \rightarrow 0 \text { as } r \rightarrow \infty
\end{gathered}
$$

and

$$
\Phi_{3} \rightarrow 0 \text { as } \rho \rightarrow \infty
$$


Equations 16 and $17 \mathrm{imply}$ that $E_{n m}=0$ and $G_{n m}=0$. Equations 3,5 , and 12 lead to

$$
\epsilon_{1}^{-1}\left[A_{n m} a^{n}+\frac{B_{n m}}{a^{n+1}}\right]=\epsilon_{3}^{-1}\left[\frac{F_{n m}}{a^{n+1}}+\frac{H_{n m}}{\rho_{a}^{n+1}}\right]
$$

where $\rho_{a}$ is $\rho$ evaluated at $r=a$, i.e.

$$
\rho_{a}^{2}=a^{2}+R^{2}-2 a R \cos \gamma \quad \rho_{a}{ }^{1}=(\mathrm{d} \rho / \mathrm{d} r)_{r=a}
$$

Let

$$
\epsilon_{a}=\left(\epsilon_{3} / \epsilon_{1}\right)
$$

With these, eq 18 may be rewritten as

$$
F_{n m}=a^{n+1}\left(\epsilon_{a} A_{n m} a^{n}+\epsilon_{a} \frac{B_{n m}}{a^{n+1}}-\frac{H_{n m}}{\rho_{a}^{n+1}}\right)
$$

Equations 3, 5, and 14 lead to

$$
n A_{n m} a^{n-1}-(n+1) \frac{B_{n m}}{a^{n+2}}=-(n+1) \frac{F_{n m}}{a^{n+2}}-(n+1) \frac{H_{n m}}{\rho_{a}^{n+2}} \rho_{a}^{1}
$$

Eliminating $F_{n m}$ from eqs 21 and 22 gives

$$
H_{n m}=\left(\frac{\epsilon_{a}}{\epsilon_{a}-\epsilon_{a}^{1}}\right)\left(\frac{\rho_{a}^{n+1}}{a^{n+1}}\right)\left[A_{n m} a^{2 n+1}\left\{\frac{n+(n+1) \epsilon_{a}}{(n+1)}\right\}+\right.
$$

where

$$
\epsilon_{a}^{1}=\frac{\epsilon_{a} a \rho_{a}^{1}}{\rho_{a}}
$$

From eqs 21 and 23, $F_{n m}$ is obtained as

$$
\begin{aligned}
F_{n m}=\left(\frac{\epsilon_{a}}{\epsilon_{a}-\epsilon_{a}^{1}}\right)\left[-A_{n m} a^{2 n+1}\left\{\frac{n+(n+1) \epsilon_{a}^{1}}{(n+1)}\right\}+\right. \\
\left.B_{n m}\left(1-\epsilon_{a}^{1}\right)\right]
\end{aligned}
$$

Similarly, eqs 4 and 5 together with eq 13 lead to

$$
\epsilon_{2}^{-1}\left(C_{n m} b^{n}+\frac{D_{n m}}{b^{n+1}}\right)=\epsilon_{3}^{-1}\left(\frac{F_{n m}}{r_{b}^{n+1}}+\frac{H_{n m}}{b^{n+1}}\right)
$$

where $r_{b}$ is $r$ evaluated at $\rho=b$.

$$
r_{b}=\left(b^{2}+R^{2}-2 b R \cos \delta\right)^{1 / 2} \text { and } r_{b}^{1}=(\mathrm{d} r / \mathrm{d} \rho)_{\rho=b}
$$

Let

$$
\epsilon_{b}=\left(\epsilon_{3} / \epsilon_{2}\right)
$$

With these, eq 26 may be rewritten as

$$
\epsilon_{b}\left[C_{n m} b^{n}+\frac{D_{n m}}{b^{n+1}}\right]=\frac{F_{n m}}{r_{b}^{n+1}}+\frac{H_{n m}}{b^{n+1}}
$$

Equations 4, 5, and 15 lead to

$$
C_{n m} n b^{n-1}-D_{n m} \frac{(n+1)}{b^{n+2}}=-F_{n m} \frac{(n+1) r_{b}^{1}}{r_{b}^{n+1}}-H_{n m} \frac{(n+1)}{b^{n+2}}
$$

Eliminating $F_{n m}$ from eqs 29 and 30 gives

$$
\begin{aligned}
& H_{n m}=\left(\frac{\epsilon_{b}}{\epsilon_{b}^{1}-\epsilon_{b}}\right)\left[C_{n m} b^{2 n+1}\left\{\frac{n+(n+1) \epsilon_{b}^{1}}{(n+1)}\right\}-\right. \\
& D_{n m}\left(1-\epsilon_{b}^{1}\right)
\end{aligned}
$$

where

$$
\epsilon_{b}^{1}=\frac{\epsilon_{b} b r_{b}^{1}}{r_{b}}
$$

Equations 29 and 31 lead to

$$
\left.\begin{array}{r}
F_{n m}=\left(\frac{r_{b}^{n+1}}{b^{n+1}}\right)\left(\frac{\epsilon_{b}}{\epsilon_{b}^{1}-\epsilon_{b}}\right)\left[-C_{n m} b^{2 n+1}\left\{\frac{n+(n+1) \epsilon_{b}}{(n+1)}\right\}+\right. \\
D_{n m}\left(1-\epsilon_{b}\right)
\end{array}\right]
$$

Eliminating $H_{n m}$ from eqs 23 and 31 and $F_{n m}$ from eqs 25 and 33 gives two equations for $A_{n m}$ and $C_{n m}$ in terms of $B_{n m}$ and $D_{n m}$. Solving for $A_{n m}$ and $C_{n m}$ gives

$$
\begin{array}{r}
A_{n m}=\frac{(n+1)}{\theta}\left[\left(1-\epsilon_{a}\right)\left(n+(n+1) \epsilon_{b}\right)-\left(\frac{a b}{\rho_{a} r_{b}}\right)^{n+1}\left(1-\epsilon_{a}^{1}\right)\right. \\
\left.\left(n+(n+1) \epsilon_{b}^{1}\right)\right] \frac{B_{n m}}{a^{2 n+1}}+\frac{(n+1)}{\theta}\left[\left(1-\epsilon_{b}^{1}\right)\left(n+(n+1) \epsilon_{b}\right)-\right. \\
\left.\quad\left(1-\epsilon_{b}\right)\left(n+(n+1) \epsilon_{b}^{1}\right)\right]\left(\frac{\epsilon_{b}}{\epsilon_{b}-\epsilon_{b}^{1}}\right)\left(\frac{\epsilon_{a}-\epsilon_{a}^{1}}{\epsilon_{a}}\right) \frac{D_{n m}}{\rho_{a}^{n+1} a^{n}}(34) \\
C_{n m}=\frac{(n+1)}{\theta}\left[\left(1-\epsilon_{a}^{1}\right)\left(n+(n+1) \epsilon_{a}\right)-\left(1-\epsilon_{a}\right)(n+(n+\right. \\
\left.\left.1) \epsilon_{a}^{1}\right)\right]\left(\frac{\epsilon_{a}}{\epsilon_{a}-\epsilon_{a}^{1}}\right)\left(\frac{\epsilon_{b}-\epsilon_{b}^{1}}{\epsilon_{b}}\right) \frac{B_{n m}}{r_{b}^{n+1} b^{n}}+\frac{(n+1)}{\theta}\left[\left(1-\epsilon_{b}\right)(n+\right. \\
\left.\left.(n+1) \epsilon_{a}\right)-\left(\frac{a b}{\rho_{a} r_{b}}\right)^{n+1}\left(1-\epsilon_{b}^{1}\right)\left(n+(n+1) \epsilon_{a}^{1}\right)\right] \frac{D_{n m}}{b^{2 n+1}}(35) \\
\theta=\left[\left(n+(n+1) \epsilon_{a}\right)\left(n+(n+1) \epsilon_{b}\right)-\left(\frac{a b}{\rho_{a} r_{b}}\right)^{n+1}(n+(n+\right. \\
\left.\left.\quad 1) \epsilon_{a}^{1}\right)\left(n+(n+1) \epsilon_{b}^{1}\right)\right](36)
\end{array}
$$

Using eqs 34 and 35 along with eqs 9-11, the Heimholtz free energy of the charge assembly can be obtained as

$$
\begin{aligned}
A=\frac{1}{2 \epsilon_{1}} \sum_{k=1}^{s} \sum_{n=0}^{\infty} \sum_{m=-n}^{+n} A_{n m} q_{k} r_{k}^{n} P_{n}^{m}\left(\cos \theta_{k}\right) \mathrm{e}^{i m \varphi_{k}}+ \\
\frac{1}{2 \epsilon_{2}} \sum_{l=1}^{t} \sum_{n=0}^{\infty} \sum_{m=-n}^{+n} C_{n m} q_{l} \rho_{l}^{n} P_{n}^{m}\left(\cos \theta_{l}\right) \mathrm{e}^{i m \varphi_{l}}
\end{aligned}
$$

Some special cases:

(a) Two charge distributions infinitely apart, $R \rightarrow \infty$. In this limit eqs 34 and 35 become

$$
\begin{aligned}
A_{n m}=\left[\frac{(n+1)\left(1-\epsilon_{a}\right)}{n+(n+1) \epsilon_{a}}\right] \frac{B_{n m}}{a^{2 n+1}} \& & C_{n m}= \\
& {\left[\frac{(n+1)\left(1-\epsilon_{b}\right)}{n+(n+1) \epsilon_{b}}\right] \frac{D_{n m}}{b^{2 n+1}} }
\end{aligned}
$$

These compare exactly with the expressions reported previously for solvation free energies (see eqs 13-19 in ref 7). With the additional condition that $n=0$, one recovers Born's expressions 
for the individual ions.

$$
A_{n m}=\left(\frac{1-\epsilon_{a}}{\epsilon_{a}}\right) \frac{B_{n m}}{a} \& C_{n m}=\left(\frac{1-\epsilon_{b}}{\epsilon_{b}}\right) \frac{D_{n m}}{b}
$$

Similarly for $n=1$, one obtains Onsager's expressions for the solvation free energy of dipoles.

(b) Two monopoles (ions) in a dielectric continuum solvent, $n=0$.

$$
A_{0}=\frac{1}{\theta_{0}}\left[\left(1-\epsilon_{a}\right) \epsilon_{b}-\left(\frac{a b}{\rho_{a} r_{b}}\right)\left(1-\epsilon_{a}^{1}\right) \epsilon_{b}^{1}\right] \frac{B_{0}}{a}+\frac{1 \epsilon_{b}}{\Theta_{0} \epsilon_{a}}\left(\epsilon_{a}-\epsilon_{a}^{1}\right) \frac{D_{0}}{\rho_{a}}
$$

$$
C_{0}=\frac{1}{\theta_{0}}\left[\left(1-\epsilon_{b}\right) \epsilon_{a}-\left(\frac{a b}{\rho_{a} r_{b}}\right)\left(1-\epsilon_{b}^{1}\right) \epsilon_{a}^{1}\right] \frac{D_{0}}{b}+\frac{1 \epsilon_{a}}{\theta_{0} \epsilon_{b}}\left(\epsilon_{b}-\epsilon_{b}^{1}\right) \frac{B_{0}}{r_{b}}
$$

$$
\begin{gathered}
\theta_{0}=\epsilon_{a} \epsilon_{b}-\left(\frac{a b}{\rho_{a} r_{b}}\right) \epsilon_{a}^{1} \epsilon_{b}^{1} \\
\gamma=0 ; \delta=0 \quad \rho_{a}=R-a ; r_{b}=R-b \quad \rho_{a}^{1}=-1 ; r_{b}^{1}=-1 \\
\epsilon_{a}^{1}=\epsilon_{a}(a /(a-R)) \quad \epsilon_{b}^{1}=\epsilon_{b}(b /(b-R)) \\
B_{0}=q_{k} \quad D_{0}=q_{l} \\
\Delta A=(1 / 2)\left[\left(1 / \epsilon_{1}\right) A_{0} q_{k}+\left(1 / \epsilon_{2}\right) C_{0} q_{l}\right]
\end{gathered}
$$

(c) Coulomb's law for two point charges imbedded in a dielectric continuum solvent. The infinities arising in the self-energies of point charges a re avoided by first taking the limit of homogeneous dielectric continuum, i.e. by equating all the different dielectric constants in the system and then letting the ionic radii go to zero. Setting $\epsilon_{1}=\epsilon_{2}=\epsilon_{3}=\epsilon$, i.e. $\epsilon_{a}=\epsilon_{b}=1$ in eqs 37 and 38 leads to

$$
\begin{aligned}
& A_{0}=\left[b^{2} R /\left\{(R-a)^{2}(R-b)^{2}\right\}\right] B_{0}+\left[R /(R-a)^{2}\right] D_{0} \\
& C_{0}=\left[a^{2} R /\left\{(R-a)^{2}(R-b)^{2}\right\}\right] D_{0}+\left[R /(R-b)^{2}\right] B_{0}
\end{aligned}
$$

The free energy expression then becomes

$$
\begin{array}{r}
\Delta A=(1 / 2 \epsilon)\left[b^{2} R /\left\{(R-a)^{2}(R-b)^{2}\right\}\right] q_{k}^{2}+(1 / 2 \epsilon)[R /(R- \\
\left.a)^{2}\right] q_{k} q_{l}+(1 / 2 \epsilon)\left[a^{2} R /\left\{(R-a)^{2}(R-b)^{2}\right\}\right] q_{l}^{2}+ \\
(1 / 2 \epsilon)\left[R /(R-b)^{2}\right] q_{k} q_{l}
\end{array}
$$

Letting $a=b=0$ in the above equation gives

$$
\Delta A=(1 / 2 \epsilon)\left[q_{l} q_{k} / R+q_{k} q_{l} / R\right]=q_{k} q_{l} /(\epsilon R)
$$

(d) Influence of a vicinal point charge on the solvation free energy of a charge. Recently Kang, Nemethy, and Scheraga ${ }^{31}$ developed a theory to estimate the Helmholtz free energy of polarization of the hydration shell of a charge due to the presence of a neighboring point charge which they identified as $\left(q_{k} q_{l}\right)$ $2 \epsilon R)$. We consider here a special case of a point charge in the vicinity of a finite-sized ion, as this proves to be a very instructive example in delineating the contributions of each part of the system.

Let $\epsilon_{b}=1$ and then $b=0$ in eqs 38 and 39

$$
\begin{gathered}
A_{0}=\left\{\left(1-\epsilon_{a}\right) / \epsilon_{a} a\right\} B_{0}+\left\{\left(1 / \epsilon_{a}\right)\left(R /(R-a)^{2}\right)\right\} D_{0} \\
C_{0}=\left\{a^{2} /\left(R(R-a)^{2}\right)\right\} D_{0}+\{1 / R\} B_{0}
\end{gathered}
$$

The Helmholtz free energy is then given as

$$
\begin{array}{r}
\Delta A=\left\{\left(1-\epsilon_{a}\right) / 2 a \epsilon_{1} \epsilon_{a}\right\} q_{k}^{2}+\left\{R /\left(2 \epsilon_{1} \epsilon_{a}(R-a)^{2}\right)\right\} q_{k} q_{l}+ \\
\left\{a^{2} /\left(2 \epsilon_{3} R(R-a)^{2}\right)\right\} q_{l}^{2}+\left\{1 / 2 \epsilon_{3} R\right\} q_{k} q_{l}
\end{array}
$$

The system in this case, it may be recalled, consists of a charge $q_{k}$ embedded in a cavity of radius $a$ and dielectric constant $\epsilon_{1}$ which is surrounded by solvent of dielectric constant $\epsilon_{3}$. There exists a point charge $q_{l}$ in the solvent region at a distance $R$ from $q_{k}$. The $\Delta A$ here represents work done for transferring both $q_{k}$ and $q_{l}$ from vacuum to solvent and bringing them together to a distance $R$ from infinity. The first term in eq 41 represents Born's self-energy for $q_{k}$. There is no such self-energy term for $q_{l}$ since it is a point charge. The second and fourth terms are the contributions of $q_{k}$ and $q_{l}$ to the interaction energy between these two charges. The fourth term is half the Coulomb energy due to the point charge $q_{l}$. The second term would reduce to the remaining half of the Coulomb energy if $a$ goes to zero. Thus, to interpret the fourth term $q_{k} q_{l} / 2 \in R$ as the polarization free energy ${ }^{31}$ due to a point charge does not appear to be justified. This is just half the Coulombic $(1 / r)$ term in any force field which takes care of charge-charge interactions. Adding this under solvation as well will result in double counting. The second term may be seen as the modified Coulomb energy due to the finite size of the ion of charge $q_{k}$. The third term then becomes the correction to the solvation (first term) free energy. In the limit of $\epsilon_{a} \rightarrow 1$ and $a \rightarrow 0$ both first and third terms vanish. A simple partitioning thus suggests that the first and third (self) terms here and in eqs 34 and 35 deal with solvation free energies while the second and fourth (cross) terms refer to the interaction energies.

Finally, the electrostatic contribution to the interaction energies between a sodium and a chloride ion are plotted in Figure 2 as a function of distance between them along with Coulombic interaction energy. Born's self-energies of the $\mathrm{Na}^{+}$ion and $\mathrm{Cl}^{-}$ ions which make a constant contribution to the curve have been subtracted out of the total free energy calculated using eq 40 . The radius of the cation and anion are taken to be 1.68 and 1.937 $\AA ;{ }^{3} \epsilon_{1}=\epsilon_{2}=1, \epsilon_{3}=80$. It may be noticed that there is an enhancement in the attractive interactions between the two ions of opposite charge relative to Coulomb's law predictions, a consequence of two opposing trends. Charge 1 polarizes solvent, and this acts back on charge 1 . Self-energy is due to this reaction field. Desolvation leads to modifications to the reaction field which increases the total free energy of the system ( $\Delta A$ becomes less negative). When depicted as in Figure 2, desolvation has the apparent effect of decreasing the net attraction between the two species relative to Coulomb's law. The second source involves charge 2 polarizing solvent, and this refraction field acts on charge 1. Modified Coulomb field originates in this. This decreases the total free energy ( $\triangle A$ becomes more negative). The contribution of this to the total free energy of the system is larger than the desolvation expense. The net result is an increased attraction between the two ions of opposite charge.

Interesting effects ensue when the distance is less than the sum of the assumed radii of the two ions. In particular, the ion pair problem changes over to a dipole problem. The minimum seen in Figure 2 is reflective of this phenomenon. Such a minimum does not and is not expected to occur with Coulomb's law. The distance at which the minimum occurs in Figure 2 is not indicative of the optimal interaction distance between the two ions. Shortrange repulsions (as may be incorporated via a $1 / r^{12}$ term) are nonelectrostatic in nature and are beyond the purview of dielectric continuum theories. The present theory may, however, be supplemented by addition of such terms for a comparison with potential of mean force data obtainable from molecular simulations and for the development of a force field which includes solvent effects. 
Charge Distributions in a Dielectric Continuum

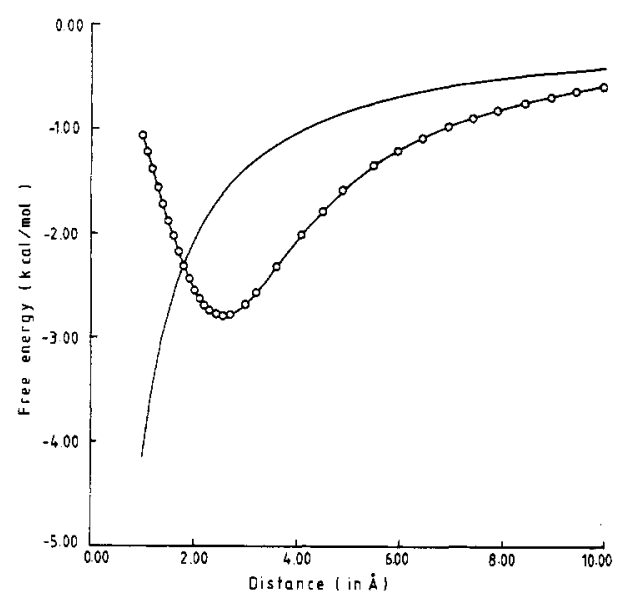

Figure 2. Electrostatic contribution to the total free energy of a system of two finite-sized ions of opposite charge imbedded in a dielectric continuum solvent, calculated using eq 40 (solid line with circles), as a function of distance between the two ions after Born's solvation energies of individual ions have been subtracted out. Interaction energies based on Coulomb's law with $\epsilon=80$ are also shown (solid line).

\section{Conclusions}

A theoretical expression (eqs 34-37) for the total free energy of a charge assembly consisting of two arbitrary charge distributions at a finite distance imbedded in a dielectric continuum solvent is obtained. The free energy arises from the field acting on each charge distribution, and this field has two components: (a) the reaction field which accounts for solvation effects and (b) the refraction field which accounts for modifications to Coulombic interactions due to the presence of dielectric discontinuities in the solution. In general, the refraction field results in an enhanced interaction strength over Coulombic interactions. Like charges repel and unlike charges attract more strongly than in the case of Coulomb's law.

Acknowledgment. The author wishes to thank Ms. Samprite Sen for verifying some of the equations, Mr. E. Rajasekaran for plotting Figure 2, and the Department of Science and Technology, India, for providing financial support.
The Journal of Physical Chemistry, Vol. 98, No. 22, 19945777

\section{References and Notes}

(1) Born, M. Z. Phys. 1920, $l, 45$.

(2) Bockris, J. O'M.; Reddy, A. K.N. Modern Electrochemistry; Plenum Press: New York, 1970; Vol. I, Chapter 2.

(3) Rashin, A. A.; Honig, B. J. Phys. Chem. 1985, 89, 5588-5593.

(4) Onsager, L. J. Am. Chem. Soc. 1936, 58, 1486.

(5) Kirkwood, J. G. J. Chem. Phys. 1934, 2, 351.

(6) Tanford, C. Physical Chemistry of Macromolecules; John Wiley \& Sons, Inc.: New York, 1961; Chapter 7. 2566.

(7) Beveridge, D. L.; Schnuelle, G. W. J. Phys. Chem. 1975, 79, 2562-

(8) Harrison, S. W.; Nolte, H. J.; Beveridge, D. L. J. Phys. Chem. 1976, $80,2580$.

(9) Gomez-Jeria, J. S.; Morales-Lagos, D. J. Phys. Chem. 1990, 94, 3790-3795.

(10) Jayaram, B.; Beveridge, D. L. Biopolymers 1988, 27, 617-627.

(11) Tanford, C.; Kirkwood, J. G. J. Am. Chem. Soc. 1957, 79, 53335339.

(12) Jayaram, B.; Mezei, M.; Beveridge, D. L. J. Am. Chem. Soc. 1988, $110,1691-1694$. 417 .

(13) Matthew, J. B. Annu. Rev. Biophys. Biophys. Chem. 1985, 14, 387-

(14) Harvey, S. C. Proteins 1989, 5, 78-92.

(15) States, D. J.; Karplus, M. J. Mol. Biol. 1987, 197, 122-130.

(16) Delepierre, M.; Dobson, C. M.; Karplus, M.; Poulsen, F. M.; States, D. J.; Wedin, R. E. J. Mol. Biol. 1987, 197, 111-122.

(17) Gilson, M.; Rashin, A.; Fine, R.; Honig, B. J. Mol. Biol. 1985, 183. 503-516.

(18) Gilson, M. K.; Honig, B. Proteins 1988, 4, 7-18.

(19) Jayaram, B.; Beveridge, D. L. J. Phys. Chem. 1990, 94, 4666-4671.

(20) Bashford, D. Curr. Opin. Struct. Biol. 1991, 1, 175-184.

(21) Jayaram, B.; Fine, R.; Sharp, K.; Honig, B. J. Phys. Chem. 1989, 93, $4320-4327$.

(22) Roux, B.; Yu, H.-A.; Karplus, M. J. Phys. Chem. 1990, 94, 46834688.

(23) Lim, C.; Bashford, D.; Karplus, M. J. Phys. Chem. 1991, 95, 56105620.

(24) Jorgensen, W. L.; Buckner, J. K.; Huston, S. E.; Rossky, P. J. J. Am. Chem. Soc. 1987, 109, 1891-1899.

(25) Rashin, A. A. J. Phys. Chem. 1989, 93, 4664-4669.

(26) Warshel, A.; Levitt, M. J. Mol. Biol. 1976, 103, 227.

(27) Warshell, A.; Russell, S. Q. Rev. Biophys. 1984, 17, 283.

(28) Oberoi, H.; Allewell, N. M. Biophys. J. 1993, 65, 48-55.

(29) Holst, M.; Saied, F. J. Comput. Chem. 1993, 14, 106-113.

(30) You, T. J.; Harvey, S. C. J. Comput. Chem. 1993, 14, 484-501.

(31) Kang, Y. K.; Nemethy, G.; Scheraga, H. A. J. Phys. Chem. 1987, $91,4109-4117$. 